\title{
Multimessenger NuEM Alerts with AMON
}

\author{
Hugo Ayala ${ }^{a, *}$ on behalf of the AMON Group, the HAWC, the IceCube and the \\ Antares Collaboration \\ (a complete list of authors can be found at the end of the proceedings) \\ a Pennsylvania State University, \\ State College, US \\ E-mail: hgayala@psu.edu
}

The Astrophysical Multimessenger Observatory Network (AMON), has developed a real-time multi-messenger alert system. The system performs coincidence analyses of datasets from gammaray and neutrino detectors, making the Neutrino-Electromagnetic (NuEM) alert channel. For these analyses, AMON takes advantage of sub-threshold events, i.e., events that by themselves are not significant in the individual detectors. The main purpose of this channel is to search for gamma-ray counterparts of neutrino events. We will describe the different analyses that make-up this channel and present a selection of recent results.

$37^{\text {th }}$ International Cosmic Ray Conference (ICRC 2021)

July 12th - 23rd, 2021

Online - Berlin, Germany

${ }^{*}$ Presenter 


\section{Multi-messenger Astrophysics}

The study of the Universe has been benefited by the improvement of our technology and analysis techniques. Recently, this has allowed us to combine the information available from different observations that focus on individual components of the physical phenomena. The most important results in the past lustrum are the detection of gravitational waves and electromagnetic radiation from the merger of a binary neutron star [1], together with the detection of high-energy neutrinos and gamma-rays from the blazar TXS 0506-056 [2].

The information given by each individual messenger - cosmic rays, electromagnetic radiation, neutrinos, and gravitational waves - help us get a general picture about the acceleration mechanisms and identification of sources that produce cosmic-rays, gamma-ray bursts, the nature of dark matter and other unsolved questions in astrophysics [See for example 3, 4].

In these contribution we present an overview of the Neutrino-Electromagnetic (NuEM) channel of the Astrophysical Multimessenger Observatory Network. This effort is based on the new paradigm of multi-messenger astrophysics, which aims at coordination between different observatories and combining independent datasets into a coincidence analysis.

\section{AMON}

The Astrophysical Multimessenger Observatory Network (AMON) is a program developed at the Pennsylvania State University. Its primary objective is to perform real-time coincidence searches of sub-threshold events of different observatories. Any statistically significant coincidence is then reported to the astrophysical community through Gamma-ray Coordinates Network (GCN, also known as the Transient Astronomy Network). AMON also stores events into its database to perform archival coincidence searches. It broadcasts individual events to GCN if the observatories providing the events consider them to be of interest to the astrophysical community. And finally, it aims to be a framework that facilitates the interaction between observatories trying to combine their datasets [5]. A list of participants can be found in the AMON webpage ${ }^{1}$.

The AMON alert system started sending alerts in 2016. Currently, AMON provides the following public alerts:

- IceCube Gold, Bronze and Cascade alerts,

- HAWC Burst-like alerts,

- NuEM channel alerts.

The notices of these alerts can be found in the GCN webpage ${ }^{2}$. The webpage also includes the now decomissioned IceCube HESE and EHE alerts.

${ }^{1}$ https://www . amon.psu.edu/amon-participants/

2https://gcn.gsfc.nasa.gov/amon.html 


\section{The NuEM Channel}

The AMON NuEM channel focuses on neutrino and high-energy photon coincidences by using sub-threshold data ${ }^{3}$ from different observatories. The main objective of this channel is to search for the sources of high-energy neutrinos with the help of high-energy gamma rays.

This search can be performed since there are physical processes where neutrinos and gamma rays are produced together. It is known that accelerated cosmic rays interact with radiation fields or interstellar matter surrounding the region around astrophysical sources. These interactions produce secondary charged and neutral pions. Charged pions mainly decay via $\pi^{+} \rightarrow \mu^{+}+v_{\mu}$, followed by the decay of the muon as $\mu^{+} \rightarrow e^{+}+v_{e}+\bar{v}_{\mu}$ and a similar process with the charge conjugate. Neutral pions decay into two gamma-ray photons, $\pi^{0} \rightarrow \gamma+\gamma$. A photohadronic interaction, i.e. a collision between a cosmic ray and a photon, will produce charged and neutral pions with probabilities of one-third and two-thirds. If the interaction is just hadronic, then the probability of producing charged and neutral pions is one-third for each type of pion [6].

The NuEM channel receives data from the IceCube [7], ANTARES [8], HAWC [9], and Fermi-LAT [10] observatories. We have performed archival coincidences analysis between: FermiANTARES [11], HAWC-IceCube [12], Fermi-IceCube [13] and HAWC-ANTARES, which preliminary results are shown in this contribution.

The general algorithm used in AMON to find and rank coincidences is as follows:

1. Set a criteria to select events which conform a coincidence. The criteria can be:

- A time window where events can arrive.

- A maximum angular distance between events.

2. Calculate a ranking statistic for the coincidence. This usually includes:

- A likelihood calculation, $\lambda(\boldsymbol{x})$, quantifying the overlap between events. Maximizing the likelihood, $\lambda_{\max }$, gives an estimate of the position of the coincidence $\boldsymbol{x}_{\max }$.

- A combination of p-values using Fisher's method [14]. The p-values can be, for example, how likely the individual event is from background, or how likely is the overlap between events just random.

3. Calculate the false-alarm rate of the coincidence as a function of the ranking statistic.

The false-alarm rate (FAR) is built by simulating random coincidences and generating a distribution of the ranking statistic. The analysis Fermi-ANTARES [11] and HAWC-IceCube [12] are, at the time of writing, running in real-time. The latency of these analyses are in the order of hours: 1 to 12 hours when data is downlinked from the Fermi-LAT instrument, and 3 to 7 hours when the data is collected by the HAWC Observatory (which corresponds to the time a point in the sky transits the detector's field of view). The calculation of the ranking statistic and the alert sending time is less than a minute. For the public real-time system we have set, with an agreement between the observatories, a threshold of FAR $<4$ per year to send alerts. Alerts are sent as notices and circulars through $\mathrm{GCN}^{4}$.

\footnotetext{
${ }^{3}$ The numerical definition of a sub-threshold event is set by each individual observatory.

${ }^{4}$ The NuEM notices are found in https://gcn.gsfc.nasa.gov/gcn/amon_nu_em_coinc_events.html
} 


\section{Results}

Table 1 shows the public alerts that appear in GCN, as well as the results of the archival coincidence searches performed so far. For the archival coincidences we show the ones that have a FAR $<1$ per year.

\begin{tabular}{|c|c|c|c|c|c|}
\hline Name & R.A. $\left[{ }^{\circ}\right]$ & Decl. $\left[{ }^{\circ}\right]$ & $\delta \theta\left[{ }^{\circ}\right]$ & FAR $\left[y r^{-1}\right]$ & Time UTC \\
\hline \multicolumn{6}{|c|}{ Real-time alerts } \\
\hline NuEM-210515A & 93.64 & 14.66 & 0.15 & 3.93 & 2021-05-15 00:20:43 \\
\hline NuEM-210515B & 93.93 & 12.51 & 0.20 & 1.90 & 2021-05-15 00:19:27 \\
\hline NuEM-210111A & 162.34 & 19.46 & 0.37 & 3.85 & 2021-01-11 13:06:41 \\
\hline NuEM-201124A & 134.99 & 7.74 & 0.23 & 2.96 & 2020-11-24 14:13:37 \\
\hline NuEM-201107A & 140.20 & 29.76 & 0.15 & 3.49 & 2020-11-07 15:55:31 \\
\hline ANTARES-Fermi 200704A & 255.42 & -34.48 & 0.43 & 0.98 & 2020-07-04 15:53:48 \\
\hline NuEM-200202A & 200.30 & 12.71 & 0.17 & 1.39 & 2020-02-02 14:07:52 \\
\hline ANTARES-Fermi 191011A & 49.96 & 18.80 & 0.40 & 1.21 & 2019-10-11 15:54:32 \\
\hline \multicolumn{6}{|c|}{ Archival Coincidences } \\
\hline ANTARES-Fermi & 248.00 & -7.7 & 0.07 & 0.09 & 2012-11-21 20:19:52 \\
\hline ANTARES-Fermi & 279.68 & -5.05 & 0.10 & 0.09 & 2014-08-05 11:13:33 \\
\hline HAWC-IceCube & 4.93 & 2.96 & 0.16 & 0.99 & 2016-12-12 04:38:41 \\
\hline HAWC-IceCube & 173.99 & 2.27 & 0.53 & 0.026 & 2018-04-12 07:54:51 \\
\hline HAWC-ANTARES & 25.6 & 25.0 & 0.2 & 0.7 & 2016-01-08 04:39:38 \\
\hline HAWC-ANTARES & 222.8 & -0.8 & 0.2 & 0.87 & 2017-09-07 01:21:22 \\
\hline HAWC-ANTARES & 85.4 & 3.4 & 0.2 & 0.41 & 2019-03-29 03:01:18 \\
\hline
\end{tabular}

Table 1: Real-time and Archival coincidences in the AMON NuEM channel. Real-time alerts are sent when the FAR is less than 4 per year. Archival coincidences have a FAR of less than 1 per year. Although the ANTARES-Fermi alerts are part of the AMON NuEM channel, the alerts are currently sent via GCN circulars instead of notices.

Follow-up observations of the real-time alerts have been made by a couple of observatories. Table 2 shows some of the instruments that submitted GCN circulars of their follow-up observations.

We have also looked at the Fermi All-sky Variability Analysis (FAVA) ${ }^{5}$ as well as the SIMBAD ${ }^{6}$ and $\mathrm{NED}^{7}$ catalogs, but no counterpart has been found so far for any of the coincidence alerts.

\section{Summary}

The multi-messenger approach has become a new paradigm to study astrophysical objects. Its advantages are that we can have a better understanding of different phenomena in the universe, such as sources of high-energy cosmic rays and neutrinos, the processes leading to gamma-ray bursts and the nature of dark matter. However, the tasks are challenging and require development of new

5https://fermi.gsfc.nasa.gov/ssc/data/access/lat/FAVA/

${ }^{6}$ http://simbad.u-strasbg.fr/simbad/

7http://ned.ipac.caltech.edu/ 


\begin{tabular}{|c|c|}
\hline Name & Followed by \\
\hline NuEM-210515A/B & ANTARES \\
NuEM-210111A & ANTARES, INTEGRAL,MAXI \\
NuEM-201124A & ANTARES \\
NuEM-201107A & Fermi-LAT \\
NuEM-200202A & MASTER, ANTARES \\
FERMI-ANTARES-191011A & MASTER \\
\hline
\end{tabular}

Table 2: Follow-up observations of the NuEM alerts published as GCN Circulars. No counterpart was found in these observations. If seen online, each alert has a link to their respective circular.

techniques as well as the cooperation between different collaborations. AMON is a framework that aims to help with these issues. As an example, we have shown here the NuEM channel, a channel that performs coincidence analyses between neutrino data and gamma-ray data. The datasets used are diverse since they come from observatories such as IceCube, ANTARES, HAWC and FermiLAT. The AMON-NuEM channel has been sending alerts to the astrophysical community since 2019. The channel is still growing, with new coincidences planned to be added to it such as the HAWC-ANTARES analysis.

\section{References}

[1] B.P. Abbott, R. Abbott, T.D. Abbott et al., Multi-messenger Observations of a Binary Neutron Star Merger, ApJ 848 (2017) L12.

[2] M.G. Aartsen, M. Ackermann, J. Adams et al., Multimessenger observations of a flaring blazar coincident with high-energy neutrino IceCube-170922A, Science 361 (2018) 8.

[3] F. Halzen, Multi-messenger astronomy: cosmic rays, gamma-rays, and neutrinos, in 21st Texas Symposium on Relativistic Astrophysics (Texas in Tuscany), 2, 2003, DOI.

[4] A. Neronov, Introduction to multi-messenger astronomy, J. Phys. Conf. Ser. 1263 (2019) 012001 [1907.07392].

[5] H.A. Ayala Solares, S. Coutu, D.F. Cowen et al., The Astrophysical Multimessenger Observatory Network (AMON): Performance and science program, Astroparticle Physics 114 (2020) 68.

[6] D. Biehl, D. Boncioli, A. Fedynitch et al., Astrophysical neutrino production and impact of associated uncertainties in photo-hadronic interactions of UHECRs, in European Physical Journal Web of Conferences, vol. 208 of European Physical Journal Web of Conferences, p. 04002, May, 2019, DOI.

[7] M.G. Aartsen, M. Ackermann, J. Adams et al., The IceCube Neutrino Observatory: Instrumentation and Online Systems, JINST P03012 (2017) 12. 
[8] M. Ageron, J. Aguilar, I.A. Samarai et al., ANTARES: The first undersea neutrino telescope, Nucl. Instrum. Methods Phys. Res. A 656 (2011) 11.

[9] A.U. Abeysekara, A. Albert, R. Alfaro et al., Observation of the Crab Nebula with the HAWC Gamma-Ray Observatory, ApJ 843 (2017) 39.

[10] M. Ackermann, M. Ajello, A. Albert et al., The Fermi Large Area Telescope on Orbit: Event Classification, Instrument Response Functions, and Calibration, ApJS 203 (2012) 4.

[11] H.A. Ayala Solares, S. Coutu, J.J. DeLaunay et al., A Search for Cosmic Neutrino and Gamma-Ray Emitting Transients in 7.3 Years of ANTARES and Fermi LAT Data, Astrophys. J. 886 (2019) 98 [1904.06420].

[12] H.A. Ayala Solares, S. Coutu, J.J. DeLaunay et al., Multimessenger Gamma-Ray and Neutrino Coincidence Alerts Using HAWC and IceCube Subthreshold Data, ApJ 906 (2021) 63 [2008. 10616].

[13] C.F. Turley, D.B. Fox, A. Keivani, J.J. DeLaunay, D.F. Cowen, M. Mostafa et al., A Coincidence Search for Cosmic Neutrino and Gamma-Ray Emitting Sources Using IceCube and Fermi LAT Public Data, Astrophys. J. 863 (2018) 64 [1802. 08165].

[14] R.A. Fisher, Statistical methods for research workers, Edinburgh, Oliver and Boyd (1938). 


\section{Full Authors List: ANTARES Collaboration}

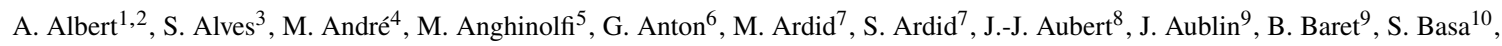
B. Belhorma ${ }^{11}$, M. Bendahman ${ }^{9}, 12$, V. Bertin ${ }^{8}$, S. Biagi ${ }^{13}$, M. Bissinger ${ }^{6}$, J. Boumaaza ${ }^{12}$, M. Bouta ${ }^{14}$, M.C. Bouwhuis ${ }^{15}$, H. Brânzaş ${ }^{16}$, R. Bruijn ${ }^{15,17}$, J. Brunner ${ }^{8}$, J. Busto ${ }^{8}$, B. Caiff ${ }^{5}$, A. Capone ${ }^{18,19}$, L. Caramete ${ }^{16}$, J. Carr ${ }^{8}$, V. Carretero ${ }^{3}$, S. Celli ${ }^{18,19}$, M. Chabab ${ }^{20}$, T. N. Chau ${ }^{9}$, R. Cherkaoui El Moursli ${ }^{12}$, T. Chiarusi ${ }^{21}$, M. Circella ${ }^{22}$, A. Coleiro ${ }^{9}$, M. Colomer-Molla ${ }^{9}, 3$, R. Coniglione ${ }^{13}$, P. Coyle ${ }^{8}$, A. Creusot ${ }^{9}$, A. F. Díaz ${ }^{23}$, G. de Wasseige ${ }^{9}$, A. Deschamps ${ }^{24}$, C. Distefano ${ }^{13}$, I. Di Palma ${ }^{18,19}$, A. Domi ${ }^{15,17}$, C. Donzaud ${ }^{9,25}$, D. Dornic ${ }^{8}$, D. Drouhin ${ }^{1,2}$, T. Eberl ${ }^{6}$, T. van Eeden ${ }^{15}$, D. van Eijk ${ }^{15}$, N. El Khayati ${ }^{12}$, A. Enzenhöfer ${ }^{8}$, P. Fermani ${ }^{18,19}$, G. Ferrara ${ }^{13}$,

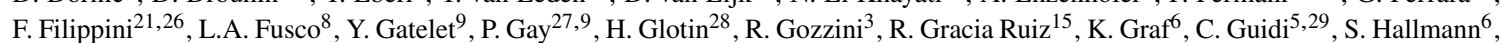
H. van Haren ${ }^{30}$, A.J. Heijboer ${ }^{15}$, Y. Hello ${ }^{24}$, J.J. Hernández-Rey ${ }^{3}$, J. Höß1 ${ }^{6}$, J. Hofestädt ${ }^{6}$, F. Huang ${ }^{8}$, G. Illuminati ${ }^{9}, 21,26$, C.W James $^{31}$, B. Jisse-Jung ${ }^{15}$, M. de Jong ${ }^{15,32}$, P. de Jong ${ }^{15}$, M. Kadler ${ }^{33}$, O. Kalekin ${ }^{6}$, U. Katz ${ }^{6}$, N.R. Khan-Chowdhury ${ }^{3}$, A. Kouchner ${ }^{9}$, I. Kreykenbohm ${ }^{34}$, V. Kulikovskiy ${ }^{5,36}$, R. Lahmann ${ }^{6}$, R. Le Breton ${ }^{9}$, D. Lefèvre ${ }^{35}$, E. Leonora ${ }^{36}$, G. Levi ${ }^{21,26}$, M. Lincetto ${ }^{8}$, D. Lopez-Coto ${ }^{37}$, S. Loucatos ${ }^{38,9}$, L. Maderer ${ }^{9}$, J. Manczak ${ }^{3}$, M. Marcelin ${ }^{10}$, A. Margiotta ${ }^{21,26}$, A. Marinelli ${ }^{39}$, J.A. MartínezMora $^{7}$, K. Melis ${ }^{15,17}$, P. Migliozzi ${ }^{39}$, A. Moussa ${ }^{14}$, R. Muller ${ }^{15}$, L. Nauta ${ }^{15}$, S. Navas ${ }^{37}$, E. Nezri ${ }^{10}$, B. O'Fearraigh ${ }^{15}$, A. Păun ${ }^{16}$, G.E. Păvălaş ${ }^{16}$, C. Pellegrino ${ }^{21,40,41}$, M. Perrin-Terrin ${ }^{8}$, V. Pestel ${ }^{15}$, P. Piattelli1 ${ }^{13}$, C. Pieterse ${ }^{3}$, C. Poirè ${ }^{7}$, V. Popa ${ }^{16}$, T. Pradier ${ }^{1}$, N. Randazzo ${ }^{36}$, S. Reck $^{6}$, G. Riccobene ${ }^{13}$, A. Romanov ${ }^{5,29}$, A. Sánchez-Losa ${ }^{3,22}$, D. F. E. Samtleben ${ }^{15,32}$, M. Sanguineti ${ }^{5,29}$ P. Sapienza ${ }^{13}$, J. Schnabel ${ }^{6}$, J. Schumann ${ }^{6}$, F. Schüssler ${ }^{38}$, M. Spurio ${ }^{21,26}$, Th. Stolarczyk ${ }^{38}$, M. Taiuti ${ }^{5,29}$, Y. Tayalati ${ }^{12}$, S.J. Tingay ${ }^{31}$, B. Vallage ${ }^{38,9}$, V. Van Elewyck ${ }^{9,41}$, F. Versari ${ }^{21,26,9}$, S. Viola ${ }^{13}$, D. Vivolo ${ }^{39,43}$, J. Wilms ${ }^{34}$, S. Zavatarelli ${ }^{5}$, A. Zegarelli ${ }^{18,19}$, J.D. Zornoza ${ }^{3}$, and J. Zúñiga ${ }^{3}$

${ }^{1}$ Université de Strasbourg, CNRS, IPHC UMR 7178, F-67000 Strasbourg, France. ${ }^{2}$ Université de Haute Alsace, F-68100 Mulhouse, France. ${ }^{3}$ IFIC - Instituto de Física Corpuscular (CSIC - Universitat de València) c/ Catedrático José Beltrán, 2 E-46980 Paterna, Valencia, Spain. ${ }^{4}$ Technical University of Catalonia, Laboratory of Applied Bioacoustics, Rambla Exposició, 08800 Vilanova i la Geltrú, Barcelona, Spain. ${ }^{5}$ INFN - Sezione di Genova, Via Dodecaneso 33, 16146 Genova, Italy. ${ }^{6}$ Friedrich-Alexander-Universität Erlangen-Nürnberg, Erlangen Centre for Astroparticle Physics, Erwin-Rommel-Str. 1, 91058 Erlangen, Germany. ${ }^{7}$ Institut d'Investigació per a la Gestió Integrada de les Zones Costaneres (IGIC) - Universitat Politècnica de València. C/ Paranimf 1, 46730 Gandia, Spain. ${ }^{8}$ Aix Marseille Univ, CNRS/IN2P3, CPPM, Marseille, France. ${ }^{9}$ Université de Paris, CNRS, Astroparticule et Cosmologie, F-75013 Paris, France. ${ }^{10}$ Aix Marseille Univ, CNRS, CNES, LAM, Marseille, France. ${ }^{11}$ National Center for Energy Sciences and Nuclear Techniques, B.P.1382, R. P.10001 Rabat, Morocco. ${ }^{12}$ University Mohammed V in Rabat, Faculty of Sciences, 4 av. Ibn Battouta, B.P. 1014, R.P. 10000 Rabat, Morocco. ${ }^{13}$ INFN - Laboratori Nazionali del Sud (LNS), Via S. Sofia 62, 95123 Catania, Italy. ${ }^{14}$ University Mohammed I, Laboratory of Physics of Matter and Radiations, B.P.717, Oujda 6000, Morocco. ${ }^{15}$ Nikhef, Science Park, Amsterdam, The Netherlands. ${ }^{16}$ Institute of Space Science, RO-077125 Bucharest, Măgurele, Romania. ${ }^{17}$ Universiteit van Amsterdam, Instituut voor Hoge-Energie Fysica, Science Park 105, 1098 XG Amsterdam, The Netherlands. ${ }^{18}$ INFN - Sezione di Roma, P.le Aldo Moro 2, 00185 Roma, Italy. ${ }^{19}$ Dipartimento di Fisica dell’Università La Sapienza, P.le Aldo Moro 2, 00185 Roma, Italy. ${ }^{20}$ LPHEA, Faculty of Science - Semlali, Cadi Ayyad University, P.O.B. 2390, Marrakech, Morocco. ${ }^{21}$ INFN - Sezione di Bologna, Viale Berti-Pichat 6/2, 40127 Bologna, Italy. ${ }^{22}$ INFN - Sezione di Bari, Via E. Orabona 4, 70126 Bari, Italy. ${ }^{23}$ Department of Computer Architecture and Technology/CITIC, University of Granada, 18071 Granada, Spain. ${ }^{24}$ Géoazur, UCA, CNRS, IRD, Observatoire de la Côte d'Azur, Sophia Antipolis, France. ${ }^{25}$ Université Paris-Sud, 91405 Orsay Cedex, France. ${ }^{26}$ Dipartimento di Fisica e Astronomia dell’Università, Viale Berti Pichat 6/2, 40127 Bologna, Italy. ${ }^{27}$ Laboratoire de Physique Corpusculaire, Clermont Université, Université Blaise Pascal, CNRS/IN2P3, BP 10448, F-63000 Clermont-Ferrand, France. ${ }^{28}$ LIS, UMR Université de Toulon, Aix Marseille Université, CNRS, 83041 Toulon, France. ${ }^{29}$ Dipartimento di Fisica dell’Università, Via Dodecaneso 33, 16146 Genova, Italy. ${ }^{30}$ Royal Netherlands Institute for Sea Research (NIOZ), Landsdiep 4, 1797 SZ 't Horntje (Texel), the Netherlands. ${ }^{31}$ International Centre for Radio Astronomy Research - Curtin University, Bentley, WA 6102, Australia. ${ }^{32}$ Huygens-Kamerlingh Onnes Laboratorium, Universiteit Leiden, The Netherlands. ${ }^{33}$ Institut für Theoretische Physik und Astrophysik, Universität Würzburg, Emil-Fischer Str. 31, 97074 Würzburg, Germany. ${ }^{34}$ Dr. Remeis-Sternwarte and ECAP, Friedrich-Alexander-Universität Erlangen-Nürnberg, Sternwartstr. 7, 96049 Bamberg, Germany. ${ }^{35}$ Mediterranean Institute of Oceanography (MIO), Aix-Marseille University, 13288, Marseille, Cedex 9, France; Université du Sud Toulon-Var, CNRS-INSU/IRD UM 110, 83957, La Garde Cedex, France. ${ }^{36}$ INFN - Sezione di Catania, Via S. Sofia 64, 95123 Catania, Italy. ${ }^{37}$ Dpto. de Física Teórica y del Cosmos \& C.A.F.P.E., University of Granada, 18071 Granada, Spain. ${ }^{38}$ IRFU, CEA, Université Paris-Saclay, F-91191 Gif-sur-Yvette, France. ${ }^{39}$ INFN - Sezione di Napoli, Via Cintia 80126 Napoli, Italy. ${ }^{40}$ Museo Storico della Fisica e Centro Studi e Ricerche Enrico Fermi, Piazza del Viminale 1, 00184, Roma. ${ }^{41}$ INFN - CNAF, Viale C. Berti Pichat 6/2, 40127, Bologna. ${ }^{42}$ Institut Universitaire de France, 75005 Paris, France. ${ }^{43}$ Dipartimento di Fisica dell'Università Federico II di Napoli, Via Cintia 80126, Napoli, Italy.

\section{Full Authors List: HAWC Collaboration}

A.U. Abeysekara ${ }^{48}$, A. Albert ${ }^{21}$, R. Alfaro ${ }^{14}$, C. Alvarez ${ }^{41}$, J.D. Álvarez ${ }^{40}$, J.R. Angeles Camacho ${ }^{14}$, J.C. Arteaga-Velázquez ${ }^{40}$, K. P. Arunbabu $^{17}$, D. Avila Rojas ${ }^{14}$, H.A. Ayala Solares ${ }^{28}$, R. Babu ${ }^{25}$, V. Baghmanyan ${ }^{15}$, A.S. Barber ${ }^{48}$, J. Becerra Gonzalez ${ }^{11}$, E. BelmontMoreno $^{14}$, D. Berley ${ }^{39}$, C. Brisbois ${ }^{39}$, K.S. Caballero-Mora ${ }^{41}$, T. Capistrán ${ }^{12}$, A. Carramiñana ${ }^{18}$, S. Casanova ${ }^{15}$, O. Chaparro-Amaro ${ }^{3}$, U. Cotti ${ }^{40}$, J. Cotzomi ${ }^{8}$, S. Coutiño de León ${ }^{18}$, E. De la Fuente ${ }^{46}$, C. de León ${ }^{40}$, L. Diaz-Cruz ${ }^{8}$, R. Diaz Hernandez ${ }^{18}$, J.C. Díaz-Vélez ${ }^{46}$, B.L. Dingus ${ }^{21}$, M. Durocher ${ }^{21}$, M.A. DuVernois ${ }^{45}$, R.W. Ellsworth ${ }^{39}$, K. Engel ${ }^{39}$, C. Espinoza ${ }^{14}$, K.L. Fan ${ }^{39}$, K. Fang ${ }^{45}$, M. Fernández 
Alonso $^{28}$, B. Fick ${ }^{25}$, H. Fleischhack ${ }^{51,11,52}$, J.L. Flores ${ }^{46}$, N.I. Fraija ${ }^{12}$, D. Garcia ${ }^{14}$, J.A. García-González ${ }^{20}$, J. L. García-Luna ${ }^{46}$, G. García-Torales ${ }^{46}$, F. Garfias ${ }^{12}$, G. Giacinti ${ }^{22}$, H. Goksu ${ }^{22}$, M.M. González ${ }^{12}$, J.A. Goodman ${ }^{39}$, J.P. Harding ${ }^{21}$, S. Hernandez ${ }^{14}$, I. $H_{e r z o g}{ }^{25}$, J. Hinton ${ }^{22}$, B. Hona ${ }^{48}$, D. Huang ${ }^{25}$, F. Hueyotl-Zahuantitla ${ }^{41}$, C.M. Hui ${ }^{23}$, B. Humensky ${ }^{39}$, P. Hüntemeyer ${ }^{25}$, A. Iriarte $^{12}$, A. Jardin-Blicq ${ }^{22,49,50}$, H. Jhee ${ }^{43}$, V. Joshi ${ }^{7}$, D. Kieda ${ }^{48}$, G J. Kunde ${ }^{21}$, S. Kunwar ${ }^{22}$, A. Lara ${ }^{17}$, J. Lee ${ }^{43}$, W.H. Lee ${ }^{12}$, D. Lennarz $^{9}$, H. León Vargas ${ }^{14}$, J. Linnemann ${ }^{24}$, A.L. Longinotti ${ }^{12}$, R. López-Coto ${ }^{19}$, G. Luis-Raya ${ }^{44}$, J. Lundeen ${ }^{24}$, K. Malone ${ }^{21}$, V. Marandon $^{22}$, O. Martinez ${ }^{8}$, I. Martinez-Castellanos ${ }^{39}$, H. Martínez-Huerta ${ }^{38}$, J. Martínez-Castro ${ }^{3}$, J.A.J. Matthews ${ }^{42}$, J. McEnery ${ }^{11}$, P. Miranda-Romagnoli ${ }^{34}$, J.A. Morales-Soto ${ }^{40}$, E. Moreno ${ }^{8}$, M. Mostafá ${ }^{28}$, A. Nayerhoda ${ }^{15}$, L. Nellen ${ }^{13}$, M. Newbold ${ }^{48}$, M.U. Nisa ${ }^{24}$, R. Noriega-Papaqui $^{34}$, L. Olivera-Nieto ${ }^{22}$, N. Omodei ${ }^{32}$, A. Peisker $^{24}$, Y. Pérez Araujo ${ }^{12}$, E.G. Pérez-Pérez ${ }^{44}$, C.D. Rho ${ }^{43}$, C. Rivière ${ }^{39}$, D. Rosa-Gonzalez ${ }^{18}$, E. Ruiz-Velasco ${ }^{22}$, J. Ryan ${ }^{26}$, H. Salazar ${ }^{8}$, F. Salesa Greus ${ }^{15,53}$, A. Sandoval ${ }^{14}$, M. Schneider ${ }^{39}$, H. Schoorlemmer ${ }^{22}$, J. Serna-Franco ${ }^{14}$, G. Sinnis ${ }^{21}$, A.J. Smith ${ }^{39}$, R.W. Springer ${ }^{48}$, P. Surajbali ${ }^{22}$, I. Taboada ${ }^{9}$, M. Tanner ${ }^{28}$, K. Tollefson ${ }^{24}$, I. Torres ${ }^{18}$, R. Torres-Escobedo $^{30}$, R. Turner ${ }^{25}$, F. Ureña-Mena ${ }^{18}$, L. Villaseñor ${ }^{8}$, X. Wang ${ }^{25}$, I.J. Watson ${ }^{43}$, T. Weisgarber ${ }^{45}$, F. Werner ${ }^{22}$, E. Willox ${ }^{39}$, J. Wood ${ }^{23}$, G.B. Yodh ${ }^{35}$, A. Zepeda ${ }^{4}$, H. Zhou ${ }^{30}$

${ }^{1}$ Barnard College, New York, NY, USA, ${ }^{2}$ Department of Chemistry and Physics, California University of Pennsylvania, California, PA, USA, ${ }^{3}$ Centro de Investigación en Computación, Instituto Politécnico Nacional, Ciudad de México, México, ${ }^{4}$ Physics Department, Centro de Investigación y de Estudios Avanzados del IPN, Ciudad de México, México, ${ }^{5}$ Colorado State University, Physics Dept., Fort Collins, CO, USA, ${ }^{6}$ DCI-UDG, Leon, Gto, México, ${ }^{7}$ Erlangen Centre for Astroparticle Physics, Friedrich Alexander Universität, Erlangen, BY, Germany, ${ }^{8}$ Facultad de Ciencias Físico Matemáticas, Benemérita Universidad Autónoma de Puebla, Puebla, México, ${ }^{9}$ School of Physics and Center for Relativistic Astrophysics, Georgia Institute of Technology, Atlanta, GA, USA, ${ }^{10}$ School of Physics Astronomy and Computational Sciences, George Mason University, Fairfax, VA, USA, ${ }^{11}$ NASA Goddard Space Flight Center, Greenbelt, MD, USA, ${ }^{12}$ Instituto de Astronomía, Universidad Nacional Autónoma de México, Ciudad de México, México, ${ }^{13}$ Instituto de Ciencias Nucleares, Universidad Nacional Autónoma de México, Ciudad de México, México, ${ }^{14}$ Instituto de Física, Universidad Nacional Autónoma de México, Ciudad de México, México, ${ }^{15}$ Institute of Nuclear Physics, Polish Academy of Sciences, Krakow, Poland, ${ }^{16}$ Instituto de Física de São Carlos, Universidade de São Paulo, São Carlos, SP, Brasil, ${ }^{17}$ Instituto de Geofísica, Universidad Nacional Autónoma de México, Ciudad de México, México, ${ }^{18}$ Instituto Nacional de Astrofísica, Óptica y Electrónica, Tonantzintla, Puebla, México, ${ }^{19}$ INFN Padova, Padova, Italy, ${ }^{20}$ Tecnologico de Monterrey, Escuela de Ingeniería y Ciencias, Ave. Eugenio Garza Sada 2501, Monterrey, N.L., 64849, México, ${ }^{21}$ Physics Division, Los Alamos National Laboratory, Los Alamos, NM, USA, ${ }^{22}$ Max-Planck Institute for Nuclear Physics, Heidelberg, Germany, ${ }^{23}$ NASA Marshall Space Flight Center, Astrophysics Office, Huntsville, AL, USA, ${ }^{24}$ Department of Physics and Astronomy, Michigan State University, East Lansing, MI, USA, ${ }^{25}$ Department of Physics, Michigan Technological University, Houghton, MI, USA, ${ }^{26}$ Space Science Center, University of New Hampshire, Durham, NH, USA, ${ }^{27}$ The Ohio State University at Lima, Lima, OH, USA, ${ }^{28}$ Department of Physics, Pennsylvania State University, University Park, PA, USA, ${ }^{29}$ Department of Physics and Astronomy, University of Rochester, Rochester, NY, USA, ${ }^{30}$ Tsung-Dao Lee Institute and School of Physics and Astronomy, Shanghai Jiao Tong University, Shanghai, China, ${ }^{31}$ Sungkyunkwan University, Gyeonggi, Rep. of Korea, ${ }^{32}$ Stanford University, Stanford, CA, USA, ${ }^{33}$ Department of Physics and Astronomy, University of Alabama, Tuscaloosa, AL, USA, ${ }^{34}$ Universidad Autónoma del Estado de Hidalgo, Pachuca, Hgo., México, ${ }^{35}$ Department of Physics and Astronomy, University of California, Irvine, Irvine, CA, USA, ${ }^{36}$ Santa Cruz Institute for Particle Physics, University of California, Santa Cruz, Santa Cruz, CA, USA, ${ }^{37}$ Universidad de Costa Rica, San José, Costa Rica, ${ }^{38}$ Department of Physics and Mathematics, Universidad de Monterrey, San Pedro Garza García, N.L., México, ${ }^{39}$ Department of Physics, University of Maryland, College Park, MD, USA, ${ }^{40}$ Instituto de Física y Matemáticas, Universidad Michoacana de San Nicolás de Hidalgo, Morelia, Michoacán, México, ${ }^{41}$ FCFM-MCTP, Universidad Autónoma de Chiapas, Tuxtla Gutiérrez, Chiapas, México, ${ }^{42}$ Department of Physics and Astronomy, University of New Mexico, Albuquerque, NM, USA, ${ }^{43}$ University of Seoul, Seoul, Rep. of Korea, ${ }^{44}$ Universidad Politécnica de Pachuca, Pachuca, Hgo, México, ${ }^{45}$ Department of Physics, University of Wisconsin-Madison, Madison, WI, USA, ${ }^{46}$ CUCEI, CUCEA, Universidad de Guadalajara, Guadalajara, Jalisco, México, ${ }^{47}$ Universität Würzburg, Institute for Theoretical Physics and Astrophysics, Würzburg, Germany, ${ }^{48}$ Department of Physics and Astronomy, University of Utah, Salt Lake City, UT, USA, ${ }^{49}$ Department of Physics, Faculty of Science, Chulalongkorn University, Pathumwan, Bangkok 10330, Thailand, ${ }^{50}$ National Astronomical Research Institute of Thailand (Public Organization), Don Kaeo, MaeRim, Chiang Mai 50180, Thailand, ${ }^{51}$ Department of Physics, Catholic University of America, Washington, DC, USA, ${ }^{52}$ Center for Research and Exploration in Space Science and Technology, NASA/GSFC, Greenbelt, MD, USA, ${ }^{53}$ Instituto de Física Corpuscular, CSIC, Universitat de València, Paterna, Valencia, Spain

\section{HAWC Acknowledgments}

We acknowledge the support from: the US National Science Foundation (NSF); the US Department of Energy Office of High-Energy Physics; the Laboratory Directed Research and Development (LDRD) program of Los Alamos National Laboratory; Consejo Nacional de Ciencia y Tecnología (CONACyT), México, grants 271051, 232656, 260378, 179588, 254964, 258865, 243290, 132197, A1-S46288, A1-S-22784, cátedras 873, 1563, 341, 323, Red HAWC, México; DGAPA-UNAM grants IG101320, IN111716-3, IN111419, IA102019, IN1 10621, IN110521; VIEP-BUAP; PIFI 2012, 2013, PROFOCIE 2014, 2015; the University of Wisconsin Alumni Research Foundation; the Institute of Geophysics, Planetary Physics, and Signatures at Los Alamos National Laboratory; Polish Science Centre grant, DEC-2017/27/B/ST9/02272; Coordinación de la Investigación Científica de la Universidad Michoacana; Royal Society - Newton Advanced Fellowship 180385; Generalitat Valenciana, grant CIDEGENT/2018/034; Chulalongkorn University's CUniverse (CUAASC) grant; Coordinación General Académica e Innovación (CGAI-UdeG), PRODEP-SEP UDG-CA-499; Institute of Cosmic Ray Research (ICRR), University of Tokyo, H.F. acknowledges support by NASA under award number 80GSFC21M0002. We also acknowledge the 
significant contributions over many years of Stefan Westerhoff, Gaurang Yodh and Arnulfo Zepeda Dominguez, all deceased members of the HAWC collaboration. Thanks to Scott Delay, Luciano Díaz and Eduardo Murrieta for technical support.

\section{Full Authors List: IceCube Collaboration}

R. Abbasi ${ }^{17}$, M. Ackermann ${ }^{59}$, J. Adams ${ }^{18}$, J. A. Aguilar ${ }^{12}$, M. Ahlers ${ }^{22}$, M. Ahrens ${ }^{50}$, C. Alispach ${ }^{28}$, A. A. Alves Jr. ${ }^{31}$, N. M. Amin ${ }^{42}$, R. An $^{14}$, K. Andeen ${ }^{40}$, T. Anderson ${ }^{56}$, G. Anton ${ }^{26}$, C. Argüelles ${ }^{14}$, Y. Ashida ${ }^{38}$, S. Axani ${ }^{15}$, X. Bai ${ }^{46}$, A. Balagopal V. ${ }^{38}$, A. Barbano ${ }^{28}$, S. W. Barwick ${ }^{30}$, B. Bastian ${ }^{59}$, V. Basu ${ }^{38}$, S. Baur ${ }^{12}$, R. Bay ${ }^{8}$, J. J. Beatty ${ }^{20,21}$, K.-H. Becker ${ }^{58}$, J. Becker Tjus ${ }^{11}$, C. Bellenghi ${ }^{27}$, S. BenZvi $^{48}$, D. Berley ${ }^{19}$, E. Bernardini ${ }^{59,}$, , D. Z. Besson ${ }^{34,61}$, G. Binder ${ }^{8,9}$, D. Bindig ${ }^{58}$, E. Blaufuss ${ }^{19}$, S. Blot ${ }^{59}$, M. Boddenberg ${ }^{1}$, F. Bontempo ${ }^{31}$, J. Borowka ${ }^{1}$, S. Böser ${ }^{39}$, O. Botner ${ }^{57}$, J. Böttcher ${ }^{1}$, E. Bourbeau ${ }^{22}$, F. Bradascio ${ }^{59}$, J. Braun ${ }^{38}$, S. Bron ${ }^{28}$, J. BrosteanKaiser $^{59}$, S. Browne ${ }^{32}$, A. Burgman ${ }^{57}$, R. T. Burley ${ }^{2}$, R. S. Busse ${ }^{41}$, M. A. Campana ${ }^{45}$, E. G. Carnie-Bronca ${ }^{2}$, C. Chen ${ }^{6}$, D. Chirkin ${ }^{38}$, K. Choi ${ }^{52}$, B. A. Clark ${ }^{24}$, K. Clark ${ }^{33}$, L. Classen ${ }^{41}$, A. Coleman ${ }^{42}$, G. H. Collin ${ }^{15}$, J. M. Conrad ${ }^{15}$, P. Coppin ${ }^{13}$, P. Correa ${ }^{13}$, D. F. Cowen $^{55}, 56$, R. Cross $^{48}$, C. Dappen ${ }^{1}$, P. Dave ${ }^{6}$, C. De Clercq ${ }^{13}$, J. J. DeLaunay ${ }^{56}$, H. Dembinski ${ }^{42}$, K. Deoskar ${ }^{50}$, S. De Ridder ${ }^{29}$, A. Desai $^{38}$, P. Desiati ${ }^{38}$, K. D. de Vries ${ }^{13}$, G. de Wasseige ${ }^{13}$, M. de With ${ }^{10}$, T. DeYoung ${ }^{24}$, S. Dharani ${ }^{1}$, A. Diaz ${ }^{15}$, J. C. Díaz-Vélez ${ }^{38}$, M.

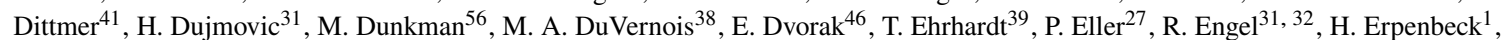
J. Evans ${ }^{19}$, P. A. Evenson ${ }^{42}$, A. R. Fazely ${ }^{7}$, S. Fiedlschuster ${ }^{26}$, A. T. Fienberg ${ }^{56}$, K. Filimonov ${ }^{8}$, C. Finley ${ }^{50}$, L. Fischer ${ }^{59}$, D. Fox $^{55}$, A. Franckowiak ${ }^{11,59}$, E. Friedman ${ }^{19}$, A. Fritz ${ }^{39}$, P. Fürst ${ }^{1}$, T. K. Gaisser ${ }^{42}$, J. Gallagher ${ }^{37}$, E. Ganster ${ }^{1}$, A. Garcia ${ }^{14}$, S. Garrappa ${ }^{59}$, L. Gerhardt $^{9}$, A. Ghadimi ${ }^{54}$, C. Glaser ${ }^{57}$, T. Glauch ${ }^{27}$, T. Glüsenkamp ${ }^{26}$, A. Goldschmidt ${ }^{9}$, J. G. Gonzalez ${ }^{42}$, S. Goswami ${ }^{54}$, D. Grant ${ }^{24}$, T. Grégoire ${ }^{56}$, S. Griswold ${ }^{48}$, M. Gündüz ${ }^{11}$, C. Günther ${ }^{1}$, C. Haack ${ }^{27}$, A. Hallgren ${ }^{57}$, R. Halliday ${ }^{24}$, L. Halve ${ }^{1}$, F. Halzen ${ }^{38}$, M. Ha

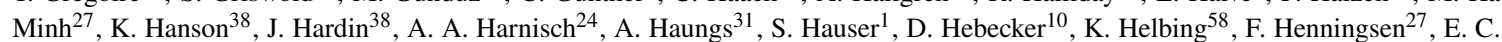
Hettinger $^{24}$, S. Hickford ${ }^{58}$, J. Hignight ${ }^{25}$, C. Hill ${ }^{16}$, G. C. Hill ${ }^{2}$, K. D. Hoffman ${ }^{19}$, R. Hoffmann ${ }^{58}$, T. Hoinka ${ }^{23}$, B. Hokanson-Fasig ${ }^{38}$, K. Hoshina ${ }^{38,62}$, F. Huang ${ }^{56}$, M. Huber ${ }^{27}$, T. Huber ${ }^{31}$, K. Hultqvist ${ }^{50}$, M. Hünnefeld ${ }^{23}$, R. Hussain ${ }^{38}$, S. In ${ }^{52}$, N. Iovine ${ }^{12}$, A. Ishihara ${ }^{16}$, M. Jansson ${ }^{50}$, G. S. Japaridze ${ }^{5}$, M. Jeong ${ }^{52}$, B. J. P. Jones ${ }^{4}$, D. Kang ${ }^{31}$, W. Kang ${ }^{52}$, X. Kang ${ }^{45}$, A. Kappes ${ }^{41}$, D. Kappesser ${ }^{39}$, T. Karg ${ }^{59}$, M. Karl ${ }^{27}$, A. Karle ${ }^{38}$, U. Katz ${ }^{26}$, M. Kauer ${ }^{38}$, M. Kellermann ${ }^{1}$, J. L. Kelley ${ }^{38}$, A. Kheirandish ${ }^{56}$, K. Kin ${ }^{16}$, T. Kintscher ${ }^{59}$, J. Kiryluk ${ }^{51}$, S. R. Klein ${ }^{8,9}$, R. Koirala ${ }^{42}$, H. Kolanoski ${ }^{10}$, T. Kontrimas $^{27}$, L. Köpke ${ }^{39}$, C. Kopper ${ }^{24}$, S. Kopper ${ }^{54}$, D. J. Koskinen ${ }^{22}$, P. Koundal ${ }^{31}$, M. Kovacevich ${ }^{45}$, M. Kowalski ${ }^{10,59}$, T. Kozynets ${ }^{22}$, E. Kun ${ }^{11}$, N. Kurahashi ${ }^{45}$, N. Lad ${ }^{59}$, C. Lagunas Gualda ${ }^{59}$, J. L. Lanfranchi ${ }^{56}$,

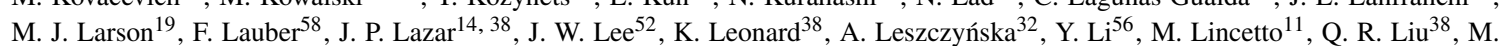
Liubarska $^{25}$, E. Lohfink ${ }^{39}$, C. J. Lozano Mariscal ${ }^{41}$, L. Lu ${ }^{38}$, F. Lucarelli28, A. Ludwig ${ }^{24}$, 35, W. Luszczak ${ }^{38}$, Y. Lyu ${ }^{8,9}$, W. Y. Ma ${ }^{59}$, J. Madsen $^{38}$, K. B. M. Mahn ${ }^{24}$, Y. Makino ${ }^{38}$, S. Mancina ${ }^{38}$, I. C. Mariş ${ }^{12}$, R. Maruyama ${ }^{43}$, K. Mase ${ }^{16}$, T. McElroy ${ }^{25}$, F. McNally ${ }^{36}$, J. V. Mead $^{22}$, K. Meagher ${ }^{38}$, A. Medina ${ }^{21}$, M. Meier ${ }^{16}$, S. Meighen-Berger ${ }^{27}$, J. Micallef $^{24}$, D. Mockler ${ }^{12}$, T. Montaruli 28 , R. W. Moore ${ }^{25}$, R. Morse ${ }^{38}$, M. Moulai ${ }^{15}$, R. Naab ${ }^{59}$, R. Nagai ${ }^{16}$, U. Naumann ${ }^{58}$, J. Necker ${ }^{59}$, L. V. Nguyễn ${ }^{24}$, H. Niederhausenn ${ }^{27}$, S. C. Nowicki ${ }^{24}$, D. R. Nygren ${ }^{9}$, A. Obertacke Pollmann ${ }^{58}$, M. Oehler ${ }^{31}$, A. Olivas ${ }^{19}$, E. O’Sullivan ${ }^{57}$, H. Pandya ${ }^{42}$, D. V. Pankova ${ }^{56}$, N. Park ${ }^{33}$, G. K. Parker ${ }^{4}$, E. N. Paudel ${ }^{42}$, L. Paul ${ }^{40}$, C. Pérez de los $\operatorname{Heros}^{57}$, L. Peters ${ }^{1}$, J. Peterson ${ }^{38}$, S. Philippen ${ }^{1}$, D. Pieloth ${ }^{23}$, S. Pieper ${ }^{58}$, M. Pittermann $^{32}$, A. Pizzuto ${ }^{38}$, M. Plum ${ }^{40}$, Y. Popovych ${ }^{39}$, A. Porcellii ${ }^{29}$, M. Prado Rodriguez ${ }^{38}$, P. B. Price ${ }^{8}$, B. Pries ${ }^{24}$, G. T. Przybylski ${ }^{9}$, C. Raab ${ }^{12}$, A. Raissi ${ }^{18}$, M. Rameez ${ }^{22}$, K. Rawlins ${ }^{3}$, I. C. Rea $^{27}$, A. Rehman ${ }^{42}$, P. Reichherzer ${ }^{11}$, R. Reimann ${ }^{1}$, G. Renzi ${ }^{12}$, E. Resconi ${ }^{27}$, S. Reusch ${ }^{59}$, W. Rhode ${ }^{23}$, M. Richman ${ }^{45}$, B. Riedel ${ }^{38}$, E. J. Roberts ${ }^{2}$, S. Robertson ${ }^{8,9}$, G. Roellinghoff ${ }^{52}$, M. Rongen ${ }^{39}$, C. Rott ${ }^{49,}{ }^{2}$, T. Ruhe ${ }^{23}$, D. Ryckbosch ${ }^{29}$, D. Rysewyk Cantu ${ }^{24}$, I. Safa ${ }^{14,38}$, J. Saffer ${ }^{32}$, S. E. Sanchez Herrera ${ }^{24}$, A. Sandrock ${ }^{23}$, J. Sandroos ${ }^{39}$, M. Santander ${ }^{54}$, S. Sarkar ${ }^{44}$, S. Sarkar ${ }^{25}$, K. Satalecka ${ }^{59}$, M. Scharf ${ }^{1}$, M. Schaufel ${ }^{1}$, H. Schieler ${ }^{31}$, S. Schindler ${ }^{26}$, P. Schlunder $^{23}$, T. Schmidt ${ }^{19}$, A. Schneider ${ }^{38}$, J. Schneider ${ }^{26}$, F. G. Schröder ${ }^{31,42}$, L. Schumacher ${ }^{27}$, G. Schwefer ${ }^{1}$, S. Sclafani ${ }^{45}$, D. Seckel ${ }^{42}$, S. Seunarine $^{47}$, A. Sharma ${ }^{57}$, S. Shefali ${ }^{32}$, M. Silva ${ }^{38}$, B. Skrzypek ${ }^{14}$, B. Smithers ${ }^{4}$, R. Snihur ${ }^{38}$, J. Soedingrekso ${ }^{23}$, D. Soldin ${ }^{42}$, C. Spannfellner $^{27}$, G. M. Spiczak ${ }^{47}$, C. Spiering ${ }^{59,} 61$, J. Stachurska ${ }^{59}$, M. Stamatikos ${ }^{21}$, T. Stanev ${ }^{42}$, R. Stein ${ }^{59}$, J. Stettner ${ }^{1}$, A. Steuer ${ }^{39}$, T. Stezelberger ${ }^{9}$, T. Stürwald ${ }^{58}$, T. Stuttard ${ }^{22}$, G. W. Sullivan ${ }^{19}$, I. Taboada ${ }^{6}$, F. Tenholt ${ }^{11}$, S. Ter-Antonyan ${ }^{7}$, S. Tilav ${ }^{42}$, F. Tischbein ${ }^{1}$,

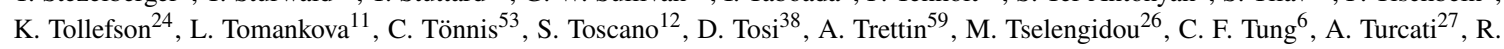
Turcotte $^{31}$, C. F. Turley ${ }^{56}$, J. P. Twagirayezu ${ }^{24}$, B. Ty ${ }^{38}$, M. A. Unland Elorrieta ${ }^{41}$, N. Valtonen-Mattila ${ }^{57}$, J. Vandenbroucke ${ }^{38}$, N. van Eijndhoven $^{13}$, D. Vannerom ${ }^{15}$, J. van Santen ${ }^{59}$, S. Verpoest ${ }^{29}$, M. Vraeghe ${ }^{29}$, C. Walck ${ }^{50}$, T. B. Watson ${ }^{4}$, C. Weaver ${ }^{24}$, P. Weigel ${ }^{15}$, A. Weindl ${ }^{31}$, M. J. Weiss ${ }^{56}$, J. Weldert ${ }^{39}$, C. Wendt ${ }^{38}$, J. Werthebach ${ }^{23}$, M. Weyrauch ${ }^{32}$, N. Whitehorn ${ }^{24}, 35$, C. H. Wiebusch ${ }^{1}$, D. R. Williams $^{54}$, M. Wolf ${ }^{27}$, K. Woschnagg ${ }^{8}$, G. Wrede ${ }^{26}$, J. Wulff ${ }^{11}$, X. W. Xu ${ }^{7}$, Y. Xu ${ }^{51}$, J. P. Yanez ${ }^{25}$, S. Yoshida ${ }^{16}$, S. Yu $^{24}$, T. Yuan $^{38}$, Z. Zhang ${ }^{51}$

${ }^{1}$ III. Physikalisches Institut, RWTH Aachen University, D-52056 Aachen, Germany

${ }^{2}$ Department of Physics, University of Adelaide, Adelaide, 5005, Australia

${ }^{3}$ Dept. of Physics and Astronomy, University of Alaska Anchorage, 3211 Providence Dr., Anchorage, AK 99508, USA

${ }^{4}$ Dept. of Physics, University of Texas at Arlington, 502 Yates St., Science Hall Rm 108, Box 19059, Arlington, TX 76019, USA

${ }^{5}$ CTSPS, Clark-Atlanta University, Atlanta, GA 30314, USA

${ }^{6}$ School of Physics and Center for Relativistic Astrophysics, Georgia Institute of Technology, Atlanta, GA 30332, USA

${ }^{7}$ Dept. of Physics, Southern University, Baton Rouge, LA 70813, USA

${ }^{8}$ Dept. of Physics, University of California, Berkeley, CA 94720, USA

${ }^{9}$ Lawrence Berkeley National Laboratory, Berkeley, CA 94720, USA

${ }^{10}$ Institut für Physik, Humboldt-Universität zu Berlin, D-12489 Berlin, Germany

${ }^{11}$ Fakultät für Physik \& Astronomie, Ruhr-Universität Bochum, D-44780 Bochum, Germany 
12 Université Libre de Bruxelles, Science Faculty CP230, B-1050 Brussels, Belgium

${ }^{13}$ Vrije Universiteit Brussel (VUB), Dienst ELEM, B-1050 Brussels, Belgium

${ }^{14}$ Department of Physics and Laboratory for Particle Physics and Cosmology, Harvard University, Cambridge, MA 02138, USA

${ }^{15}$ Dept. of Physics, Massachusetts Institute of Technology, Cambridge, MA 02139, USA

${ }^{16}$ Dept. of Physics and Institute for Global Prominent Research, Chiba University, Chiba 263-8522, Japan

${ }^{17}$ Department of Physics, Loyola University Chicago, Chicago, IL 60660, USA

${ }^{18}$ Dept. of Physics and Astronomy, University of Canterbury, Private Bag 4800, Christchurch, New Zealand

${ }^{19}$ Dept. of Physics, University of Maryland, College Park, MD 20742, USA

${ }^{20}$ Dept. of Astronomy, Ohio State University, Columbus, OH 43210, USA

${ }^{21}$ Dept. of Physics and Center for Cosmology and Astro-Particle Physics, Ohio State University, Columbus, OH 43210, USA

22 Niels Bohr Institute, University of Copenhagen, DK-2100 Copenhagen, Denmark

${ }^{23}$ Dept. of Physics, TU Dortmund University, D-44221 Dortmund, Germany

24 Dept. of Physics and Astronomy, Michigan State University, East Lansing, MI 48824, USA

${ }^{25}$ Dept. of Physics, University of Alberta, Edmonton, Alberta, Canada T6G 2E1

${ }^{26}$ Erlangen Centre for Astroparticle Physics, Friedrich-Alexander-Universität Erlangen-Nürnberg, D-91058 Erlangen, Germany

${ }^{27}$ Physik-department, Technische Universität München, D-85748 Garching, Germany

${ }^{28}$ Département de physique nucléaire et corpusculaire, Université de Genève, CH-1211 Genève, Switzerland

${ }^{29}$ Dept. of Physics and Astronomy, University of Gent, B-9000 Gent, Belgium

${ }^{30}$ Dept. of Physics and Astronomy, University of California, Irvine, CA 92697, USA

${ }^{31}$ Karlsruhe Institute of Technology, Institute for Astroparticle Physics, D-76021 Karlsruhe, Germany

${ }^{32}$ Karlsruhe Institute of Technology, Institute of Experimental Particle Physics, D-76021 Karlsruhe, Germany

33 Dept. of Physics, Engineering Physics, and Astronomy, Queen's University, Kingston, ON K7L 3N6, Canada

${ }^{34}$ Dept. of Physics and Astronomy, University of Kansas, Lawrence, KS 66045, USA

${ }^{35}$ Department of Physics and Astronomy, UCLA, Los Angeles, CA 90095, USA

36 Department of Physics, Mercer University, Macon, GA 31207-0001, USA

${ }^{37}$ Dept. of Astronomy, University of Wisconsin-Madison, Madison, WI 53706, USA

${ }^{38}$ Dept. of Physics and Wisconsin IceCube Particle Astrophysics Center, University of Wisconsin-Madison, Madison, WI 53706, USA

${ }^{39}$ Institute of Physics, University of Mainz, Staudinger Weg 7, D-55099 Mainz, Germany

${ }^{40}$ Department of Physics, Marquette University, Milwaukee, WI, 53201, USA

${ }^{41}$ Institut für Kernphysik, Westfälische Wilhelms-Universität Münster, D-48149 Münster, Germany

42 Bartol Research Institute and Dept. of Physics and Astronomy, University of Delaware, Newark, DE 19716, USA

${ }^{43}$ Dept. of Physics, Yale University, New Haven, CT 06520, USA

${ }^{44}$ Dept. of Physics, University of Oxford, Parks Road, Oxford OX1 3PU, UK

45 Dept. of Physics, Drexel University, 3141 Chestnut Street, Philadelphia, PA 19104, USA

${ }^{46}$ Physics Department, South Dakota School of Mines and Technology, Rapid City, SD 57701, USA

${ }^{47}$ Dept. of Physics, University of Wisconsin, River Falls, WI 54022, USA

${ }^{48}$ Dept. of Physics and Astronomy, University of Rochester, Rochester, NY 14627, USA

${ }^{49}$ Department of Physics and Astronomy, University of Utah, Salt Lake City, UT 84112, USA

50 Oskar Klein Centre and Dept. of Physics, Stockholm University, SE-10691 Stockholm, Sweden

${ }^{51}$ Dept. of Physics and Astronomy, Stony Brook University, Stony Brook, NY 11794-3800, USA

52 Dept. of Physics, Sungkyunkwan University, Suwon 16419, Korea

${ }^{53}$ Institute of Basic Science, Sungkyunkwan University, Suwon 16419, Korea

${ }^{54}$ Dept. of Physics and Astronomy, University of Alabama, Tuscaloosa, AL 35487, USA

55 Dept. of Astronomy and Astrophysics, Pennsylvania State University, University Park, PA 16802, USA

${ }^{56}$ Dept. of Physics, Pennsylvania State University, University Park, PA 16802, USA

${ }^{57}$ Dept. of Physics and Astronomy, Uppsala University, Box 516, S-75120 Uppsala, Sweden

${ }^{58}$ Dept. of Physics, University of Wuppertal, D-42119 Wuppertal, Germany

${ }^{59}$ DESY, D-15738 Zeuthen, Germany

${ }^{60}$ Università di Padova, I-35131 Padova, Italy

${ }^{61}$ National Research Nuclear University, Moscow Engineering Physics Institute (MEPhI), Moscow 115409, Russia

${ }^{62}$ Earthquake Research Institute, University of Tokyo, Bunkyo, Tokyo 113-0032, Japan

\section{IceCube Acknowledgments}

USA - U.S. National Science Foundation-Office of Polar Programs, U.S. National Science Foundation-Physics Division, U.S. National Science Foundation-EPSCoR, Wisconsin Alumni Research Foundation, Center for High Throughput Computing (CHTC) at the University of Wisconsin-Madison, Open Science Grid (OSG), Extreme Science and Engineering Discovery Environment (XSEDE), Frontera computing project at the Texas Advanced Computing Center, U.S. Department of Energy-National Energy Research Scientific Computing Center, Particle astrophysics research computing center at the University of Maryland, Institute for Cyber-Enabled Research at Michigan State University, and Astroparticle physics computational facility at Marquette University; Belgium - Funds for Scientific Research (FRS-FNRS and FWO), FWO Odysseus and Big Science programmes, and Belgian Federal Science Policy Office (Belspo); 
Germany - Bundesministerium für Bildung und Forschung (BMBF), Deutsche Forschungsgemeinschaft (DFG), Helmholtz Alliance for Astroparticle Physics (HAP), Initiative and Networking Fund of the Helmholtz Association, Deutsches Elektronen Synchrotron (DESY), and High Performance Computing cluster of the RWTH Aachen; Sweden - Swedish Research Council, Swedish Polar Research Secretariat, Swedish National Infrastructure for Computing (SNIC), and Knut and Alice Wallenberg Foundation; Australia - Australian Research Council; Canada - Natural Sciences and Engineering Research Council of Canada, Calcul Québec, Compute Ontario, Canada Foundation for Innovation, WestGrid, and Compute Canada; Denmark - Villum Fonden and Carlsberg Foundation; New Zealand Marsden Fund; Japan - Japan Society for Promotion of Science (JSPS) and Institute for Global Prominent Research (IGPR) of Chiba University; Korea - National Research Foundation of Korea (NRF); Switzerland - Swiss National Science Foundation (SNSF); United Kingdom - Department of Physics, University of Oxford. 